\title{
Characterization of subsurface overlapping cylindrical inclusions by infrared Thermography
}

by R. Celorrio*, M. Costa**, S.M. Shibli**, E. Apiñaniz ${ }^{* * *}$, A. Mendioroz ${ }^{* * *}$, and A. Salazar*

*Departamento de Matemática Aplicada, Universidad de Zaragoza, Campus Río Ebro, Edificio Torres Quevedo, 50018 Zaragoza, Spain

**Instituto de Física, Universidade de São Paulo, Caixa Postal 66318, 05315-970, São Paulo, SP, Brazil

***Departamento de Física Aplicada I, Escuela Técnica Superior de Ingeniería, Universidad del País Vasco,

Alameda Urquijo s/n, 48013 Bilbao, Spain

In the last years, several works on the propagation of thermal waves in materials with cylindrical $[1,2]$ and spherical [3] inclusions have been published. These works demonstrate that infrared thermography is a valuable tool to study the thermal properties of multilayer samples, fiber reinforced composites and particulate reinforced composites. In these works it is assumed that there is no interconnection between the inclusions. However, in some real materials with periodic arrangements of cylindrical or spherical particles, a certain degree of connection between them clearly appears. Such is the case of inverse opals, which consist of a regular arrangement of spherical overlapping voids surrounded by solid walls [4]. On the other hand, in the case of composites with a high fiber's volume fraction, unexpected high values of the effective thermal conductivity in the direction perpendicular to the fibers have been found. This is probably due to a direct contact between fibers, favoring heat propagation directly through the highly thermal conducting fibers. This arrangement reduces the high thermal resistance resulting from the in-series highly conducting fiber-poor conducting matrix stacks. Therefore to study their thermal properties we need to know how heat propagates through these connected arrays of cylinders and spheres.

Here, we study the scattering of thermal waves by overlapping cylinders. An analytical solution of the surface temperature of an opaque material, illuminated by a modulated light beam, with two overlapping parallel cylinders buried beneath the surface has been found. The method is based on the expansion of plane and cylindrical thermal waves in series of Bessel and Hankel functions. Measurements on calibrated samples using infrared lock-in thermography have been performed in order to confirm the validity of the model.

Plane illumination

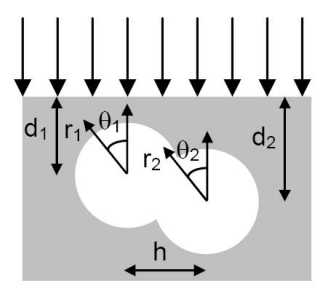

(a)

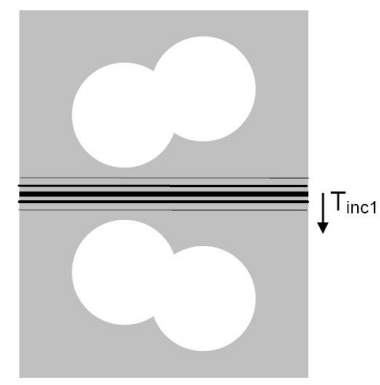

(b)

Fig. 1. (a) Geometry of the problem. (b) Equivalent geometry after applying the image theorem.

The geometry of the problem we are dealing with is shown in figure 1. The material is assumed to be semiinfinite and opaque and its surface is illuminated by a flat light beam modulated at a frequency $f(\omega=2 \pi f)$. Two infinite and parallel cylinders of radius a1 and a2, are buried at depths $\mathrm{d} 1$ and $\mathrm{d} 2$. The plane thermal wave generated at the sample surface is scattered by the two overlapping cylinders. Due to the adiabatic boundary condition at the sample surface, the real geometry shown in figure 1 (a) can be analyzed by considering the configuration shown in figure $1(\mathrm{~b})$. The effect of the wall is accounted for via the introduction of two image cylinders and an image plane thermal wave. Accordingly, the temperature at any point of the material (TM) can be written as the superposition of the incident plane thermal wave (Tinc1) with the scattered waves coming out from the two real cylinders and from their images:

$$
T_{M}\left(r_{1}, \theta_{1}\right)=T_{\text {inc } 1}\left(r_{1}, \theta_{1}\right)+\sum_{j=1}^{4} T_{\text {scatt }}^{(j)}\left(r_{1}, \theta_{1}\right)
$$

The incident plane thermal wave can be expanded in series of cylindrical waves by using the Bessel functions of the first kind (Jm) [5]:

$$
T_{i n c 1}\left(r_{1}, \theta_{1}\right)=T_{o} e^{-i q_{M}\left(x_{1}-d_{1}\right)}=T_{o} e^{i q_{M} d_{1}} \sum_{m=-\infty}^{\infty} i^{-m} J_{M}\left(q_{M} r_{1}\right) e^{i m \theta_{1}}
$$


[6]:

while the scattered cylindrical waves can be expanded in series of Hankel functions of the first kind $(\mathrm{Hm})$

$$
T_{\text {scatt }}^{(j)}\left(r_{j}, \theta_{j}\right)=\sum_{m=-\infty}^{\infty} A_{m}^{(j)} H_{M}\left(q_{M} r_{j}\right) e^{i m \theta_{j}} \quad j=1, \ldots ., 4
$$
functions [7]:

On the other hand, the temperature inside the cylinders can be expanded in terms of the ordinary Bessel

$$
T_{c}^{(j)}\left(r_{j}, \theta_{j}\right)=\sum_{m=-\infty}^{\infty} B_{m}^{(j)} J_{M}\left(q_{c} r_{j}\right) e^{i m \theta_{j}} \quad j=1,2
$$

Here $\mathrm{q}=(1+\mathrm{i}) / \mu$ is the thermal wave vector, being $\mu=(D / \pi f)^{0.5}$ the thermal diffusion length and $\mathrm{D}$ the thermal diffusivity. Subindexes $M$ and $C$ stand for matrix and cylinders respectively. The solution of the temperature field in the material and in the two cylinders requires the knowledge of the $4 \mathrm{~m}$ constants $A_{m}^{(1)}, A_{m}^{(2)}, B_{m}^{(1)}$, and $B_{m}^{(2)}$ They can be obtained from the $4 \mathrm{~m}$ equations provided by the boundary conditions (temperature and heat flux continuity) at the surface of each cylinder:

$$
T_{c}^{(j)}\left(r_{j}=a_{j}\right)=\left\{\begin{array}{ll}
T_{M}\left(r_{j}=a_{j}\right) & \text { for the non-overlapping region } \\
T_{c}^{(i)}\left(r_{j}=a_{j}\right) & \text { for the overlapping region }
\end{array} \quad i j=1,2 \quad i \neq j\right.
$$

$$
K_{c} \nabla T_{c}^{(j)}\left(r_{j}=a_{j}\right)=\left\{\begin{array}{ll}
K_{M} \nabla T_{M}\left(r_{j}=a_{j}\right) & \text { for the non-overlapping region } \\
K_{c} \nabla T_{M}^{(i)}\left(r_{j}=a_{j}\right) & \text { for the overlapping region }
\end{array} \quad i j=1,2 i \neq j\right.
$$

In figure 2 we show the calculations of the amplitude and phase of the surface temperature of an opaque material with two cylinders of the same size $(\mathrm{a} 1=\mathrm{a} 2=1 \mathrm{~mm})$, buried at the same depth $(\mathrm{d} 1=\mathrm{d} 2=1.2 \mathrm{~mm})$. We analyze the effect of varying the lateral distance $h$ : continuous line $(h=2.2 \mathrm{~mm}$, i.e. non-overlapping cylinders), dashed line ( $h=1.8 \mathrm{~mm}$, i.e. small overlapping) and dotted line $(\mathrm{h}=1 \mathrm{~mm}$, i.e. high overlapping). Calculations have been performed for good thermal conducting cylinders inside a worse thermal conducting matrix: $\mu \mathrm{M}=0.5$ $\mathrm{mm}$ and $\mu \mathrm{c}=4 \mathrm{~mm}$. Both amplitude and phase are normalized to the surface temperature far away from the cylinders. As can be seen, as the distance between cylinders is reduced the resolution of the two cylinders becomes more difficult.
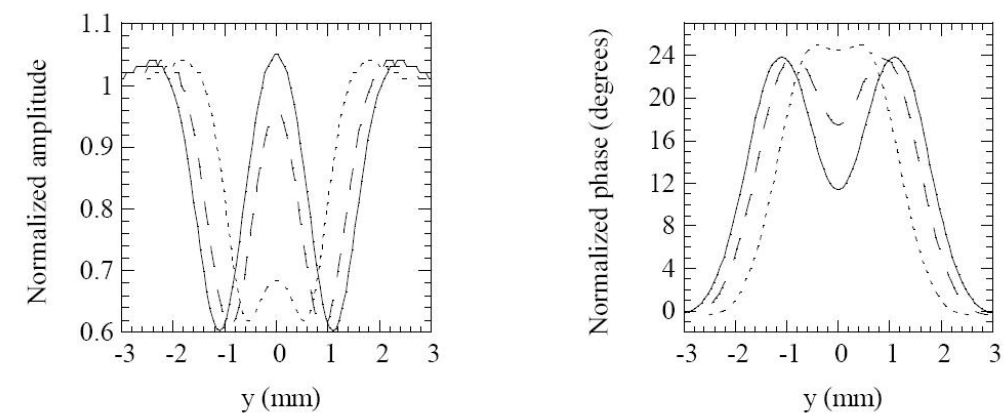

Fig. 2. Amplitude and phase of the surface temperature of two buried cylinders.

We have prepared calibrated overlapping cylinders by drilling overlapping holes in graphite samples. Measurements of the surface temperature using infrared lock-in thermography confirm the validity of the model.

This work has been supported by the Ministerio de Educación y Ciencia through research grant No MAT2005-02999 and by the Diputación Foral de Vizcaya through research grant No DIPE06/06.

\section{REFERENCES}

[1] J.M. Terrón, A. Salazar, and A. Sánchez-Lavega. Journal of Applied Physics, 91 (2002) 1087.

[2] M.L. Rapún and F.J. Sayas. Archives of Computational Methods in Engineering, 14 (2007) 3.

[3] F. Garrido, and A. Salazar. Journal of Applied Physics, 95 (2004) 140.

[4] A.E. Aliev, S.B. Lee, R.H. Baughman, and A.A. Zakhidov. Journal of Luminescence, 125 (2007) 11.

[5] G.B. Arfken, and H.J. Weber. Mathematical Methods for Physicists. Academic Press (2005).

[6] P.M. Morse, and K.U. Ingard. Theoretical Acoustics. McGraw-Hill (1968).

[7] P.M. Morse, and H. Feshbach. Methods of Theoretical Physics. McGraw-Hill (1953) 